\title{
Automated Evaluation of Crithidia luciliae Based Indirect Immunofluorescence Tests: A Novel Application of the EUROPattern-Suite Technology
}

\author{
Stefan Gerlach, Kai Affeldt, Lena Pototzki, Christopher Krause, \\ Jörn Voigt, Johanna Fraune, and Kai Fechner \\ Institute for Experimental Immunology, EUROIMMUN, Seekamp 31, 23560 Lübeck, Germany \\ Correspondence should be addressed to Kai Fechner; k.fechner@euroimmun.de
}

Received 3 April 2015; Accepted 14 June 2015

Academic Editor: Xuan Zhang

Copyright (C) 2015 Stefan Gerlach et al. This is an open access article distributed under the Creative Commons Attribution License, which permits unrestricted use, distribution, and reproduction in any medium, provided the original work is properly cited.

Systemic lupus erythematosus (SLE) is a severe rheumatic autoimmune disease with various clinical manifestations. Anti-dsDNA antibodies are an important immunological hallmark of SLE and their occurrence represents a major criterion for the diagnosis. Among the commonly applied test systems for determination of anti-dsDNA antibodies, the indirect immunofluorescence test (IIFT) using the flagellated kinetoplastida Crithidia luciliae is considered to be highly disease specific at moderate sensitivity. Since IIFT, however, is claimed to be affected by subjective interpretation and a lack of standardization, there has been an increasing demand for automated pattern interpretation of immunofluorescence reactions in recent years. Corresponding platforms are already available for evaluation of anti-nuclear antibody (ANA) IIFT on HEp-2 cells, the recommended "gold standard" for ANA screening in the diagnosis of various systemic rheumatic autoimmune diseases. For one of these systems, the "EUROPattern-Suite" computer-aided immunofluorescence microscopy (CAIFM), automated interpretation of microscopic fluorescence patterns was extended to the Crithidia luciliae based anti-dsDNA IIFT.

\section{Introduction}

For diagnosis of systemic lupus erythematosus (SLE), determination of autoantibodies is of significant diagnostic importance $[1,2]$. Among these, antibodies against double-stranded DNA (anti-dsDNA) play a major role. Their presence constitutes an important immunological criterion for the diagnosis of SLE as stated by the American College of Rheumatology in 1982 [3]. A more recent approach by the Systemic Lupus Collaborating Clinics to revise and validate the American College of Rheumatology SLE classification criteria approved anti-dsDNA as a major serological feature of SLE, considering them as very specific and a marker of disease activity and kidney involvement [4]. Accordingly, studies in mice and humans provided evidences for a role of anti-dsDNA in the pathogenesis of lupus nephritis [2, 5-9].

Information on the prevalence of anti-dsDNA in SLE varies between studies, ranging from $30 \%$ to $98 \%[2,10]$. The application of different laboratory tests is one cause which contributes to this deviation $[11,12]$. The most common methods for the detection of anti-dsDNA are enzyme-linked immunosorbent assays (ELISA), radio immunoassays (RIA, e.g., Farr assays and PEG assays) and Crithidia luciliae indirect immunofluorescence tests (CLIFT) [13]. It is hypothesized that each of these detects individual, yet overlapping, subgroups of anti-dsDNA revealing divergent properties (e.g., avidity, structural specificity) and, of particular interest, different clinical associations $[10,14]$. Classical anti-dsDNA ELISA is accepted as the most sensitive but often less specific method for SLE diagnostics. Through modifications of the applied DNA substrates and their linkage to the test wells, an increase in diagnostic accuracy of the ELISA for SLE could be achieved in recent years $[15,16]$. Nevertheless, consistency between different ELISA kits seems to be limited [12]. Therefore, primary test results usually require confirmation by a second assay such as Farr immunoassay and/or CLIFT, both of which are regarded as highly disease specific, detecting only antibody subpopulations with a high positive predictive 
value for SLE [10, 12, 14, 17-19]. Since RIA employ radioactive elements, CLIFT is commonly considered as more applicable confirmatory test system in the clinical routine of SLE diagnostics [20].

CLIFT utilizes the protist Crithidia luciliae as substrate, taking advantage of its kinetoplast, a network of tightly packed dsDNA within a large mitochondrion. In contrast to the nucleus, the kinetoplast contains fewer proteins and thus allows a more selective detection of anti-dsDNA antibodies [21]. Sensitivities of the assay have been reported to range from around $30 \%$ to nearly $60 \%$ at very high disease specificities of typically above $95 \%$ [12, 14-16]. Therefore, CLIFT is appreciated as a useful tool to support the diagnosis of SLE and its discrimination from other diseases.

A limitation of CLIFT however is-as generally applies to the procedure of indirect immunofluorescence tests (IIFT) the manual read-out of fluorescence signals and its subjective interpretation which lead to a high intra- and interlaboratory variability [12, 22-25]. Great efforts, therefore, have been made in previous years to develop automated solutions enabling optimal image acquisition as well as objective and standardized evaluation of immunofluorescence results, especially in the major field of ANA diagnostics [24, 26, 27]. IIFT on HEp-2 cells still is the recommended "gold standard" for ANA determination [28-30]. Thus, several commercial platforms for automated immunofluorescence microscopy have been developed and validated [31-37]. The automation was shown to greatly contribute to standardization and facilitation of ANA HEp-2 IIFT interpretation. Particularly with regard to positive/negative discrimination, the new systems achieved a very high consensus with manual result interpretation [38-41].

Among these platforms, the EUROPattern-Suite (Euroimmun AG, Lübeck, Germany) is a system for computeraided immunofluorescence microscopy, combining several hardware and software modules for fully automated image acquisition and evaluation. It performs reliable discrimination of positive and negative ANA HEp-2 (and HEp-20-10) IIFT results. Additionally it provides the option of automated and accurate recognition of several single as well as mixed ANA patterns and titer estimation [32, 39, 40, 42]. Results and corresponding images are displayed within a userfriendly graphical interface (GUI) which allows interactive revision and requires final validation by the professional operator. Thus, the system can reach full compliance with visual immunofluorescence microscopy in terms of result interpretation. In comparison to classical microscopy, the EUROPattern-Suite requires less hands-on effort and is much more resistant to human error. A detailed description of the technology and its associated laboratory management system EUROLabOffice is provided by Krause et al. [42].

Here, we present the first data on automated fluorescence interpretation of CLIFT using the EUROPattern-Suite. Only very few systems have been described in this context in the literature so far $[34,43,44]$.

\section{Material and Methods}

2.1. Human Sera. A panel of 569 consecutive human sera which were sent to an immunological reference laboratory
(Lübeck, Germany) for routine anti-dsDNA screening as well as 100 sera of healthy blood donors were examined. Samples were blinded for the analysis, which was carried out in accordance to the ethical guideline stated in the Declaration of Helsinki (1964).

\subsection{Crithidia luciliae Indirect Immunofluorescence Test (CLIFT).} Indirect immunofluorescence on Crithidia luciliae was performed using the Crithidia luciliae (anti-dsDNA) EUROPattern kit following the manufacturer's instruction (Euroimmun AG, Lübeck, Germany). One slide contains 10 reaction areas, each provided with one biochip $(2 \times 2 \mathrm{~mm}$ fragments of coated cover slip glued into the reaction fields), coated with cells of the protist. Slides were manually incubated and washed with the help of the TITERPLANE technique. Samples were applied at a dilution of 1:10 in PBS-Tween. Fluorescein isothiocyanate (FITC-) labeled goat anti-human IgG was used for green fluorescent staining. Antiserum was supplied with Evans blue, used for red fluorescent counterstaining of the cells.

2.3. Evaluation of Anti-dsDNA Antibodies. A focused image of each biochip on the incubated slides was automatically taken by the EUROPattern fluorescence microscope (see description below). Images were then interpreted in terms of sample positivity/negativity, once automatically by the EUROPattern software and, in a parallel approach, visually by two experts working independently of each other and without notice of software results. Disagreements between visual results were decided by a third opinion. Anti-dsDNA titers of $\geq 1$ : 10 were considered positive.

2.4. Description of the System. A detailed description of the general EUROPattern-Suite hardware and software composition is provided in [32, 42].

A new classification software has been specifically developed for the recognition and interpretation of anti-dsDNA on Crithidia luciliae: Two images per biochip, one in the green and one in the red fluorescence channel of the microscope, are taken at a 400 -fold magnification, using the 40x microscope objective. On average, this magnification leads to the recording of 30 cells per image. Autofocusing is performed using transmitted light to avoid fluorescence bleaching. The underlying algorithm for subsequent fine adjustments has been adapted to the needs of Crithidia luciliae image acquisition, resulting in a focused fluorescence image at a resolution of $2,448 \times 2,048$ pixels within 18 seconds. Thus, a slide containing 10 biochips is processed in less than three minutes. Since the EUROPattern microscope is equipped with two cameras, corresponding images in the green (specific FITC fluorescence signal) and the red (Evans blue counterstaining) fluorescence channel are taken at the same time.

The following image classification process operates asynchronously, meaning that the software already provides the first results for interactive verification while the microscope is still running. The procedure incorporates multiple steps which are performed in sequential order (Figure 1). 

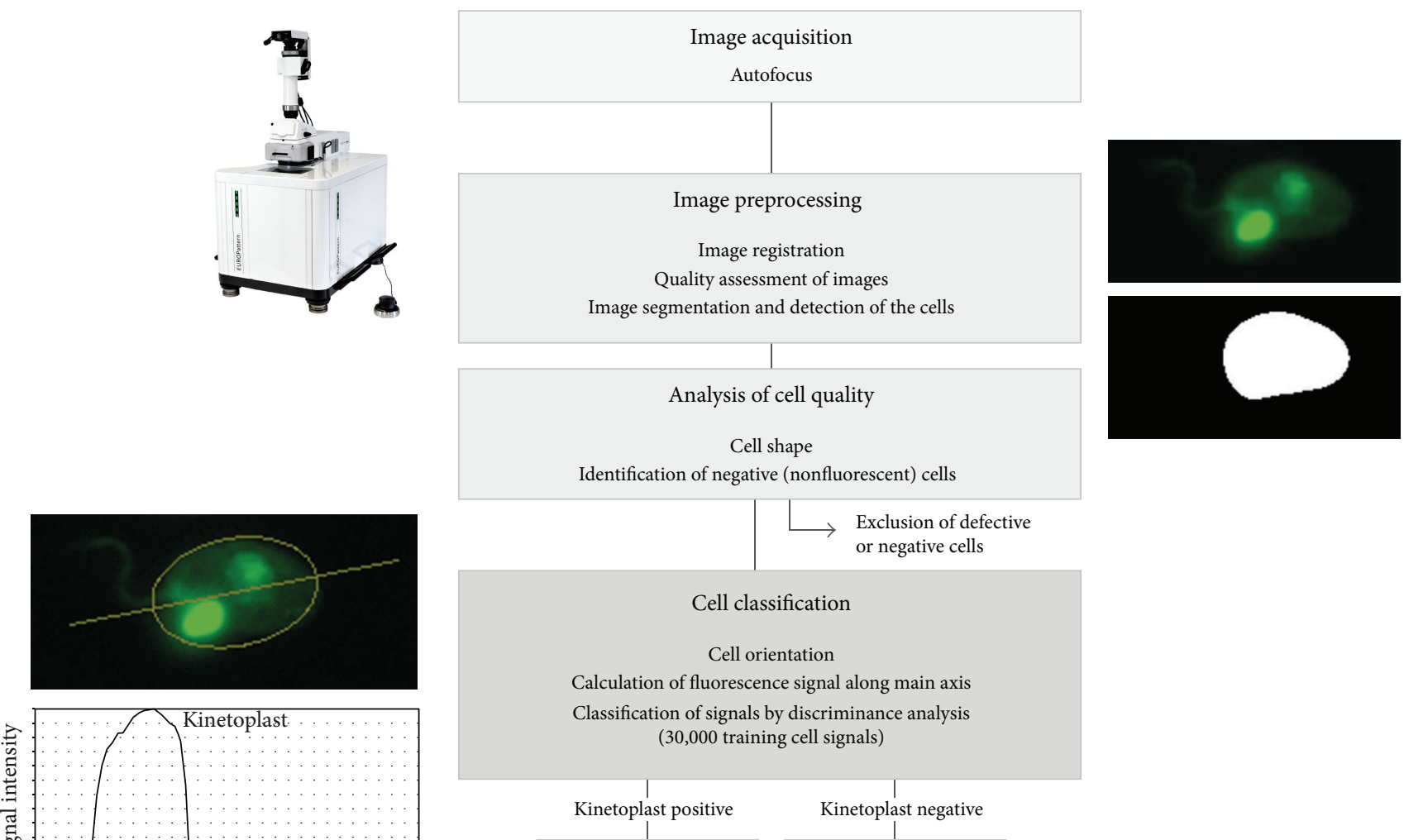

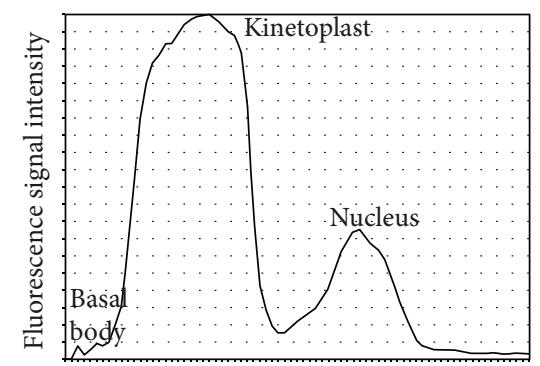

Cell axis from back to tip
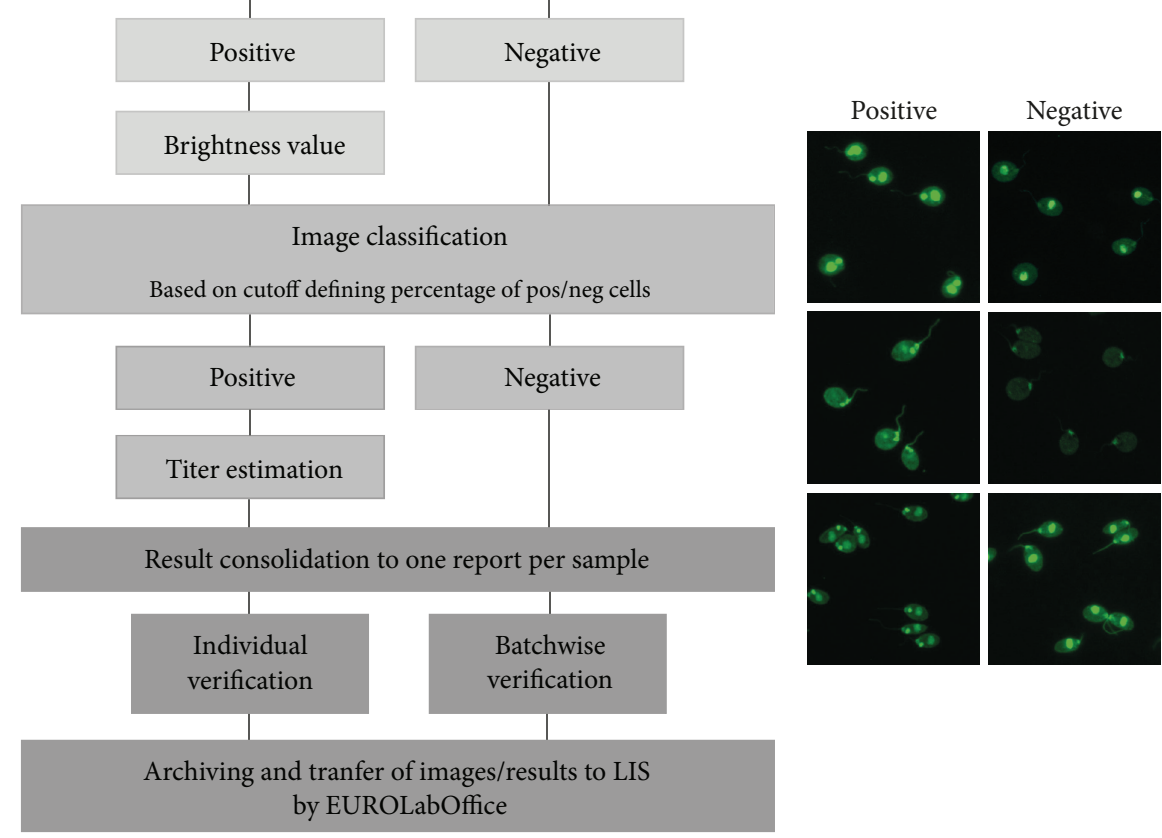

FIGURE 1: Flowchart of EUROPattern-Suite algorithm for computer-aided immunofluorescence microscopy of Crithidia luciliae IFT applied for the detection of antibodies against dsDNA.

(i) Image Preprocessing. Images of the green and red channel are loaded to memory and a perfect overlay of each image pair is generated which is important for subsequent image segmentation. Images are then analyzed regarding focal imprecision and potential incubation artefacts by application of specific software algorithms incorporating convolutional filter. Controlling the image sharpness is performed in the red fluorescence channel in which every cell can be detected. This strategy allows reliable discrimination between images of anti-dsDNA negative cells and images taken out of focus. As assessed by an internal visual validation, less than $0.1 \%$ of acquired images may reveal some degree of focal inaccuracy. Afterwards, image segmentation and precise detection of any cell are again performed in the red fluorescence 
channel. Adaptive thresholding techniques are used to mask as well as select every cell by means of connected components.

(ii) Analyzing Cell Quality. Each cell mask is now examined in terms of shape characteristics and potential defects, such as size, ellipse form, aspect ratio, or defects of the convex hull. Defective cells are excluded from subsequent evaluation. Additionally, negative cells are identified by application of a thresholdbased algorithm which assesses the brightness of the complete cell. If all cells are "dark," the complete image is set to negative and not processed any further.

(iii) Cell Classification. For this purpose, the kinetoplasts of the recognized and fluorescent cells need to be identified. This is achieved by the following steps. First, the software determines the orientation for each cell. Afterwards, a discrete and normalized signal is generated along the calculated main axis, based on mathematical measures such as mean value and standard deviation. Due to the set order of the cellular organelles (basal body, kinetoplast, and nucleus), the signal encodes their specific fluorescence intensities and even discloses the case of an organelle being absent. In a second step, extracted normalized signals are now classified by means of a discriminance analysis based on a reference training database. This database contains images of 30,000 incubated cells which have been acquired from incubations in the reference laboratory or in the context of validation studies. Each of the training images has been labelled by experts with specific information indicating the presence or absence of any fluorescent cell organelle. As a result of this classification, every cell is assigned as either positive or negative according to the fluorescence status of the kinetoplast. Furthermore, a brightness value is extracted from the kinetoplast fluorescence signal.

(iv) Image Classification. Images are classified into antidsDNA positive or negative, based on a configurable cutoff which defines the required percentage of positive cells within the image. Titer estimation per image is achieved by aggregating the cell brightness values and transforming them into an antibody titer (given in configurable titer steps). A confidence value is calculated which corresponds to the probability of the proposed results. Classifications and titer proposals concerning different dilutions of the same sample are merged into one final result which is given adjacent to the corresponding images within the GUI at the computer screen. The software generated result has to be verified by the physician and confirmed by one mouse click. This verification can be performed batchwise for negative samples and is executed one by one for positive samples.

\section{Results}

Beneficial automation of IIFT evaluation in a diagnostic laboratory requires reliable interpretation of the fluorescent images in clinical routine, encompassing high accuracy of
TABLE 1: Comparison of software-generated and visual positive/negative classification including 669 analyzed samples.

\begin{tabular}{lcccc}
\hline \multirow{2}{*}{669} & \multicolumn{4}{c}{ Visual evaluation } \\
& & Positive & Negative & $\Sigma$ \\
\hline \multirow{2}{*}{ EUROPattern } & Positive & $\mathbf{7 3}$ & 19 & 92 \\
& Negative & 0 & $\mathbf{5 7 7}$ & 577 \\
& $\Sigma$ & 73 & 596 & 669 \\
\hline Sensitivity & $100 \%$ & & & \\
Specificity & $96.8 \%$ & & & \\
Accuracy & $97.2 \%$ & & & \\
\hline
\end{tabular}

Concordant results are presented in bold font, differing results in italic font.

software derived results compared to classical visual inspection by an expert and efficient operation and processing of the system. In case of CLIFT, this means accurate discrimination between positive and negative anti-dsDNA findings according to the presence or absence of a fluorescent Crithidia luciliae kinetoplast (Figure 2) within a time- and labor-saving evaluation work flow.

Therefore, the performance of the EUROPattern-Suite (CLIFT classification software) has been validated on the basis of a large number of consecutive sera which have been tested for anti-dsDNA with the help of a commercial CLIFT kit (Euroimmun AG, Lübeck, Germany). Images of all samples were automatically taken by the EUROPattern microscope. Every image revealed a high focal precision, as determined by the software algorithm (see Section 2), thus all of them were suited for subsequent evaluation.

3.1. Positive/Negative Classification (All Samples). Images of 669 tested samples in total were evaluated in terms of positivity/negativity either automatically by EUROPattern software or visually by two experts for CLIFT interpretation (Table 1). Visual inspection yielded 73 anti-dsDNA positive and 596 anti-dsDNA negative sera. Software generated results were $100 \%$ accurate with respect to positivity implying that the system likewise recognized the same 73 anti-dsDNA positive samples. Of note, $93 \%$ of the EUROPattern titer proposals for positive samples were concordant to visual estimations within the scope of reproducibility of immunofluorescence assays (+/-1 titer level, data not shown). Out of the 596 antidsDNA negative samples, the software correctly recognized 577 cases. The remaining 19 samples were determined to be negative by eye but positive by the software. Overall results correlate to a $100 \%$ sensitive and $96.8 \%$ specific determination of anti-dsDNA on Crithidia luciliae by the EUROPatternSuite. Compared to visual microscopy, overall accuracy of the software was as high as $97.2 \%$.

\section{Discussion}

The need for standardization and automation of IIFT is tremendous in all fields of autoimmune diagnostics in order to ensure objective antibody determination. The technological progress has generated automatic solutions for incubation and processing of slides and, primarily concerning ANA diagnostics on HEp-2 cells, even imaging and evaluation 


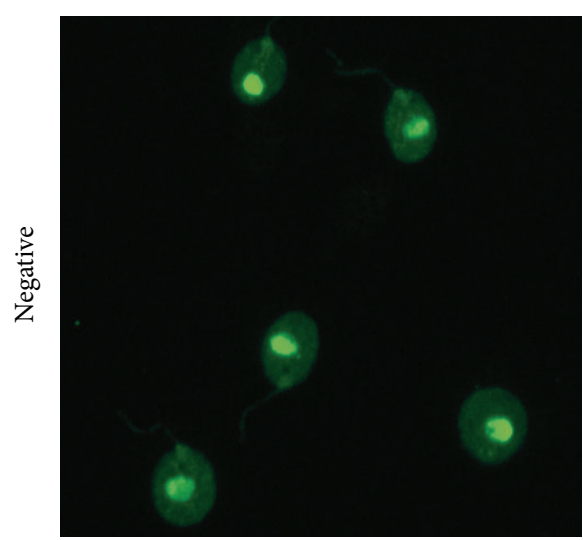

(a)

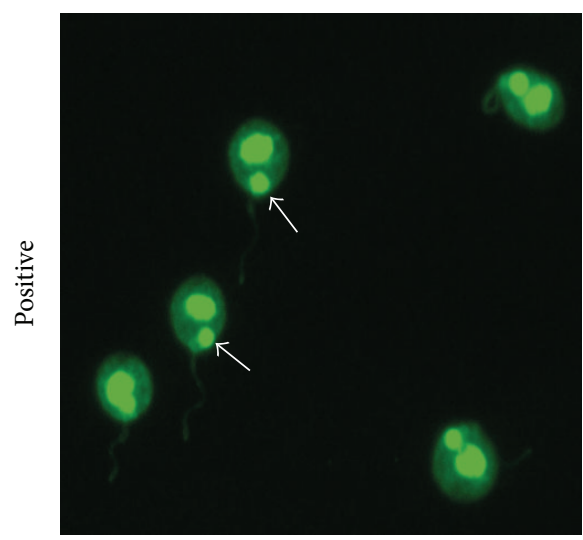

(d)

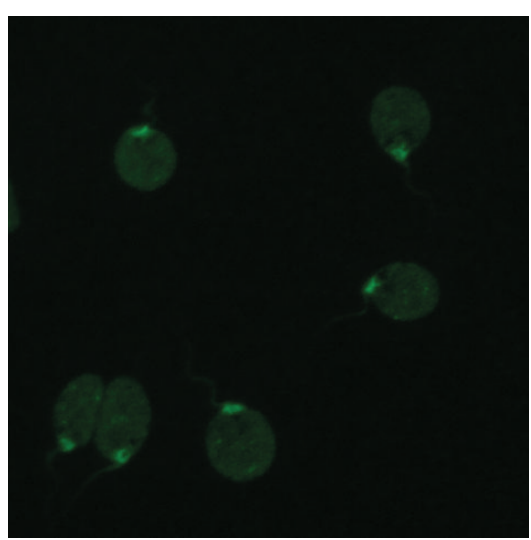

(b)

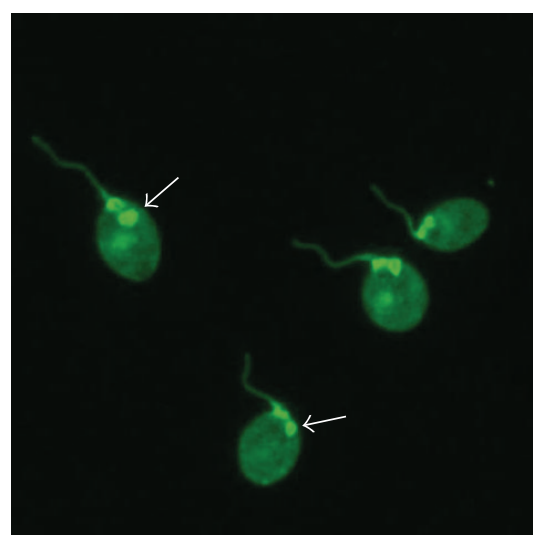

(e)

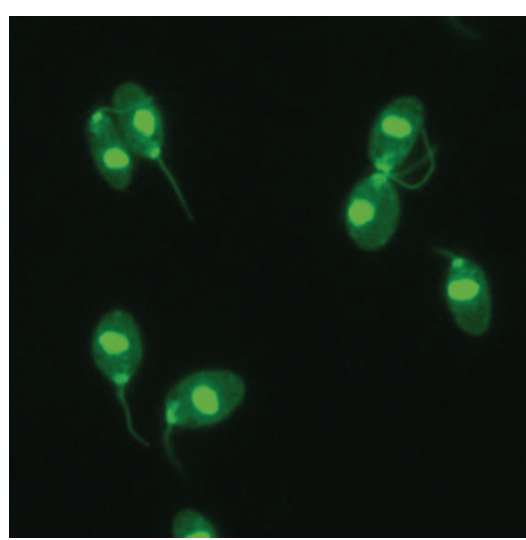

(c)

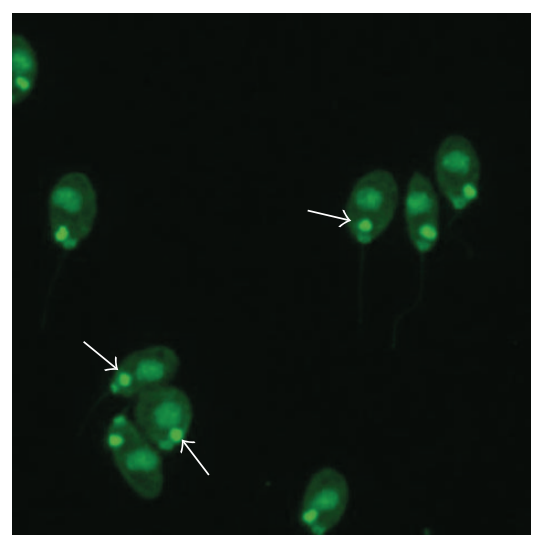

(f)

FIGURE 2: Immunofluorescence patterns on Crithidia luciliae revealing the absence or presence of antibodies against dsDNA. Samples are determined as anti-dsDNA negative if the kinetoplasts of the cells do not fluoresce, irrespective of fluorescent nuclei (a), basal bodies (b), or both (c). Samples are determined as anti-dsDNA positive as soon as the kinetoplasts reveal fluorescence signals above a given threshold (see arrows) which may be accompanied by additional fluorescence of the nuclei (d), the basal body (e), or both (f).

of fluorescence results [39]. Several platforms for IIFT automation, which differ in certain features (e.g., throughput, walk-away times, and DNA counterstain) and applications (e.g., pattern classification, number of recognized patterns, recognition of different substrates, and titer estimation), have been developed and launched for commercial use, facilitating and standardizing ANA IIFT diagnostics in numerous laboratories worldwide [38-42]. Efforts though have been majorly focused on the recognition and interpretation of the HEp-2 cell substrate. Only few commercial systems, among these the EUROPattern-Suite, provide the expanded option of automated evaluation of other substrates such as Crithidia luciliae [34, 42], which represents the most important IIFT substrate to detect anti-dsDNA in the context of diagnosing SLE. More comprehensive reports on an automated platform to support Crithidia luciliae image classification have been published for a noncommercial computer-aided-diagnosis (CAD) system previously $[43,44]$.

Similarly to the EUROPattern-Suite technology, the presented CAD system applies a multistep classification approach. Since the optical elements of the EUROPattern microscope, camera resolution, optical magnification, and cell density are adjusted to each other with high-precision, a single image is sufficient for accurate classification of one well/biochip. This leads to high performance and sample throughput in routine diagnostics. The CAD system requires classification of three to five images to classify one well, resulting in one additional classification step compared to the EUROPattern-Suite approach (see Figure 1 in this work and Figure 3 in [43]).

A threshold-based preclassification step is included in both system architectures identifying and separating either negative images (CAD) or negative cells (EUROPattern). The cell-based preclassification of the EUROPattern-Suite is robust regarding image artefacts, avoiding inclusion of false positive images into the further classification process. Single cell classification, based on extracted features, is then applied in the CAD as well as in the EUROPattern system to decide whether single cells and finally the image is positive. However, about 8 cells are recorded per image by the CAD system [44] while the average number of cells within an image taken by the EUROPattern-Suite is about 30. 
We did not find any information whether a counterstaining of the cells is used by the CAD system. In the case of the EUROPattern-Suite, Evans blue counterstaining is applied for reliable evaluation of image sharpness and for robust cell segmentation. An option of titer prediction, as provided by EUROPattern, has not been described for the CAD system $[43,44]$.

Within the scope of this study, the performance of the EUROPattern-Suite in daily clinical routine has been validated. 569 consecutive sera which have been submitted to a reference laboratory for routine anti-dsDNA screening and 100 samples from healthy blood donors were examined by CLIFT to determine anti-dsDNA. Automatically generated results by the EUROPattern-Suite were compared to results obtained through classic visual inspection by two independent experts. This comparison revealed a high accuracy of the automated evaluation strategy. In total, $97.2 \%$ of the samples were equally classified. An agreement of $91 \%$ was previously reported for another commercial system [34]. Rare cases of inaccuracy in our study exclusively concerned the negative class, as determined by the experts' visual examination, which were proposed to be positive by the software (specificity $96.8 \%)$. These false positive classifications were primarily caused by an intensive fluorescence of the basal bodies within the analyzed cells, which was misleading for the software. Further efforts are now focused on the elimination of this inaccuracy. Nevertheless, the physician may rely on the system detecting all positive samples (100\% sensitivity), meaning negative results require no closer inspection anymore. Within the GUI, these are displayed in a list, sorted by brightness and classification confidence, and can be easily verified in batches directly at the computer screen. Since the physician's verification is required for generation of the final result, this batchwise processing significantly enhances the overall efficiency of the system. The physician can now focus on the necessary review of the positive samples. Regarding these, the software allows inspection of the images as well as corrections (if required) and validation of the results one after another. Misleading result proposals, therefore, should be counterbalanced by the physician's revision.

The results support the idea of the EUROPatternSuite standardizing and facilitating SLE diagnostics through automation of CLIFT interpretation. The system is less prone to human interpretation error and functions consistently and time-effectively allowing a long walk-away time [42]. At the same time, it reached concordance with experts of CLIFT interpretation in the vast majority of cases tested during this study. All images and proposed results are displayed within the clearly arranged GUI, which is analogously designed to the ANA evaluation screen and likewise incorporated into the superordinate laboratory management software called EUROLabOffice (ELO) [42]. The results may be revised and need to be validated by the physician in a last step to generate an official end result and a diagnosis directly at the computer screen. Thus, the system has the potential to reach $100 \%$ accuracy with respect to visual immunofluorescence microscopy.

Beyond that, ELO manages the complete communication between any standard laboratory information system and the different workstations which can be found in a diagnostic laboratory (e.g., ELISA, IIFT, and immunoblot). All results which have been validated by the physician are reported to ELO and will be merged into one concluding report concerning one sample. The report is integrated into the patient's data history which is accessible via ELO. The integrated database harbors enough memory to additionally save worklists and analytical data, for example, IIFT and immunoblot images. Thus, ELO ensures automated data transfer avoiding errors by manual input, increases laboratory efficiency by taking over several organizational tasks, and supersedes the classical laboratory paper archive.

4.1. Perspectives. Further applications of the EUROPatternSuite are currently under validation or development. These include the already available option of automated immunofluorescence evaluation of antibodies against neutrophil granulocytes (ANCA) using human cell (granulocytes) substrate. The software performs positive/negative classification as well as ANCA pattern discrimination as majorly required in vasculitis diagnostics. Automated documentation and image acquisition of numerous tissues (e.g., liver, kidney, stomach, esophagus, small intestine, heart, and neuronal tissue), which are provided as cryosections on the biochips, is also feasible. With their help, organ as well as nonorgan specific antibodies (e.g., anti-mitochondrial antibodies (AMA), antibodies against epithelial membranes (EMA), epidermal basement membrane or desmosomes, anti-heart muscle-antibodies, or antibodies against various neuronal proteins) may be detected which play an important role in the diagnostics of various other autoimmune diseases.

\section{Conclusion}

Automated determination of antibodies against dsDNA using the EUROPattern-Suite for computer-aided immunofluorescence microscopy on Crithidia luciliae represents a new tool in SLE diagnostics. Validation of this system revealed $100 \%$ sensitivity and high specificity (96.8\%) for recognition and discrimination of anti-dsDNA positive and negative samples compared to visual inspection by experts of CLIFT evaluation. To raise the system's efficiency, negative samples can be verified in batches while positive samples are individually controlled and verified by an expert. In combination with the superordinate laboratory management system EUROLabOffice, the EUROPattern-Suite, therefore, enables a more standardized interpretation of CLIFT and a reduction of laboratory workload.

\section{Conflict of Interests}

Stefan Gerlach, Kai Affeldt, Lena Pototzki, Christopher Krause, Jörn Voigt, Johanna Fraune, and Kai Fechner are employees of EUROIMMUN AG.

\section{Acknowledgments}

The authors thank Dr. Konstantin Ens, Melanie Hahn, Maick Danckwardt, and Christian Feirer for their assistance and 
participation in the development of the EUROPattern-Suite technology and Dr. Jacqueline Gosink for careful and critical reading of the paper.

\section{References}

[1] "Guidelines for referral and management of systemic lupus erythematosus in adults. American College of Rheumatology Ad Hoc Committee on Systemic Lupus Erythematosus Guidelines," Arthritis \& Rheumatism, vol. 42, no. 9, pp. 1785-1796, 1999.

[2] E. Cozzani, M. Drosera, G. Gasparini, and A. Parodi, "Serology of lupus erythematosus: correlation between immunopathological features and clinical aspects," Autoimmune Diseases, vol. 2014, Article ID 321359, 13 pages, 2014.

[3] E. M. Tan, A. S. Cohen, J. F. Fries et al., "The 1982 revised criteria for the classification of systemic lupus erythrematosus," Arthritis \& Rheumatism, vol. 25, no. 11, pp. 1271-1277, 1982.

[4] M. Petri, A.-M. Orbai, G. S. Alarcõn et al., "Derivation and validation of the systemic lupus international collaborating clinics classification criteria for systemic lupus erythematosus," Arthritis and Rheumatism, vol. 64, no. 8, pp. 2677-2686, 2012.

[5] A. J. G. Swaak, J. Groenwold, L. A. Aarden, L. W. S. van Eps, and E. W. Feltkamp, "Prognostic value of anti-dsDNA in SLE," Annals of the Rheumatic Diseases, vol. 41, no. 4, pp. 388-395, 1982.

[6] E. J. ter Borg, G. Horst, E. J. Hummel, P. C. Limburg, and C. G. M. Kallenberg, "Measurement of increases in anti-doublestranded DNA antibody levels as a predictor of disease exacerbation in systemic lupus erythematosus. A long-term, prospective study," Arthritis \& Rheumatism, vol. 33, no. 5, pp. 634-643, 1990.

[7] M. C. J. van Bruggen, C. Kramers, M. N. Hylkema, R. J. T. Smeenk, and J. H. M. Berden, "Significance of anti-nuclear and anti-extracellular matrix autoantibodies for albuminuria in murine lupus nephritis; A longitudinal study on plasma and glomerular eluates in MRL/l mice," Clinical \& Experimental Immunology, vol. 105, no. 1, pp. 132-139, 1996.

[8] S. Yung and T. M. Chan, "Autoantibodies and resident renal cells in the pathogenesis of lupus nephritis: getting to know the unknown," Clinical and Developmental Immunology, vol. 2012, Article ID 139365, 13 pages, 2012.

[9] S. Yung and T. M. Chan, "Anti-DNA antibodies in the pathogenesis of lupus nephritis-the emerging mechanisms," Autoimmunity Reviews, vol. 7, no. 4, pp. 317-321, 2008.

[10] W. Egner, "The use of laboratory tests in the diagnosis of SLE," Journal of Clinical Pathology, vol. 53, no. 6, pp. 424-432, 2000.

[11] K. H. Kim, J. Y. Han, J. M. Kim, S. W. Lee, and W. T. Chung, "Clinical significance of ELISA positive and immunofluorescence negative anti-dsDNA antibody," Clinica Chimica Acta, vol. 380, no. 1-2, pp. 182-185, 2007.

[12] T. R. Chiaro, K. W. Davis, A. Wilson, B. Suh-Lailam, and A. E. Tebo, "Significant differences in the analytic concordance between anti-dsDNA IgG antibody assays for the diagnosis of systemic lupus erythematosus-implications for interlaboratory testing," Clinica Chimica Acta, vol. 412, no. 11-12, pp. 1076-1080, 2011.

[13] A.-M. Rouquette and C. Desgruelles, "Detection of antibodies to dsDNA: an overview of laboratory assays," Lupus, vol. 15, no. 7, pp. 403-407, 2006.
[14] K. Haugbro, J. C. Nossent, T. Winkler, Y. Figenschau, and O. P. Rekvig, "Anti-dsDNA antibodies and disease classification in antinuclear antibody positive patients: the role of analytical diversity," Annals of the Rheumatic Diseases, vol. 63, no. 4, pp. 386-394, 2004.

[15] R. Biesen, C. Dähnrich, A. Rosemann et al., "Anti-dsDNA-NcX ELISA: dsDNA-loaded nucleosomes improve diagnosis and monitoring of disease activity in systemic lupus erythematosus," Arthritis Research and Therapy, vol. 13, no. 1, article R26, 2011.

[16] A. Antico, S. Platzgummer, D. Bassetti, N. Bizzaro, R. Tozzoli, and D. Villalta, "Diagnosing systemic lupus erythematosus: new-generation immunoassays for measurement of antidsDNA antibodies are an effective alternative to the Farr technique and the Crithidia luciliae immunofluorescence test," Lupus, vol. 19, no. 8, pp. 906-912, 2010.

[17] B. B. Suh-Lailam, T. R. Chiaro, K. Wayne Davis, A. R. Wilson, and A. E. Tebo, "Evaluation of a high avidity anti-dsDNA igG enzyme-linked immunosorbent assay for the diagnosis of systemic lupus erythematosus," International Journal of Clinical and Experimental Pathology, vol. 4, no. 8, pp. 748-754, 2011.

[18] R. J. T. Smeenk, "Detection of antibodies to dsDNA: current insights into its relevance," Clinical and Experimental Rheumatology, vol. 20, no. 3, pp. 294-300, 2002.

[19] H. C. Nossent and O. P. Rekvig, "Is closer linkage between systemic lupus erythematosus and anti-double-stranded DNA antibodies a desirable and attainable goal?" Arthritis Research and Therapy, vol. 7, no. 2, pp. 85-87, 2005.

[20] R. Tozzoli, N. Bizzaro, E. Tonutti et al., "Guidelines for the laboratory use of autoantibody tests in the diagnosis and monitoring of autoimmune rheumatic diseases," American Journal of Clinical Pathology, vol. 117, no. 2, pp. 316-324, 2002.

[21] L. A. Aarden, E. R. de Groot, and T. E. W. Feltkamp, "Immunology of DNA. III. Crithidia luciliae, a simple substrate for the determination of anti-dsDNA with the immunofluorescence technique," Annals of the New York Academy of Sciences, vol. 254, pp. 505-515, 1975.

[22] N. Bizzaro, R. Tozzoli, E. Tonutti et al., "Variability between methods to determine ANA, anti-dsDNA and anti-ENA autoantibodies: a collaborative study with the biomedical industry," Journal of Immunological Methods, vol. 219, no. 1-2, pp. 99107, 1998.

[23] D. Roggenbuck, D. Reinhold, R. Hiemann, U. Anderer, and K. Conrad, "Standardized detection of anti-ds DNA antibodies by indirect immunofluorescence-a new age for confirmatory tests in SLE diagnostics," Clinica Chimica Acta, vol. 412, no. 21-22, pp. 2011-2012, 2011.

[24] A. Rigon, P. Soda, D. Zennaro, G. Iannello, and A. Afeltra, "Indirect immunofluorescence in autoimmune diseases: assessment of digital images for diagnostic purpose," Cytometry Part B: Clinical Cytometry, vol. 72, no. 6, pp. 472-477, 2007.

[25] M. J. Fritzler, “The antinuclear antibody test: last or lasting gasp?" Arthritis and Rheumatism, vol. 63, no. 1, pp. 19-22, 2011.

[26] U. Sack, S. Knoechner, H. Warschkau, U. Pigla, F. Emmrich, and M. Kamprad, "Computer-assisted classification of HEp2 immunofluorescence patterns in autoimmune diagnostics," Autoimmunity Reviews, vol. 2, no. 5, pp. 298-304, 2003.

[27] R. Hiemann, T. Büttner, T. Krieger, D. Roggenbuck, U. Sack, and K. Conrad, "Challenges of automated screening and differentiation of non-organ specific autoantibodies on HEp-2 cells," Autoimmunity Reviews, vol. 9, no. 1, pp. 17-22, 2009. 
[28] American College of Rheumatology, American College of Rheumatology Position Statement: Methodology of Testing for Antinuclear Antibodies, 2009, http://www.rheumatology.org.

[29] W. Stöcker and W. Schlumberger, "Antinukleäre Antikörper," in Lexikon der Medizinischen Laboratoriumsdiagnostik, A. M. Gressner and T. Arndt, Eds., vol. 2, Springer Medizin, Heidelberg, Germany, 2013.

[30] N. Agmon-Levin, J. Damoiseaux, C. Kallenberg et al., "International recommendations for the assessment of autoantibodies to cellular antigens referred to as anti-nuclear antibodies," Annals of the Rheumatic Diseases, vol. 73, no. 1, pp. 17-23, 2014.

[31] K. Egerer, D. Roggenbuck, R. Hiemann et al., "Automated evaluation of autoantibodies on human epithelial-2 cells as an approach to standardize cell-based immunofluorescence tests," Arthritis Research \& Therapy, vol. 12, no. 2, article R40, 2010.

[32] J. Voigt, C. Krause, E. Rohwäder et al., "Automated indirect immunofluorescence evaluation of antinuclear autoantibodies on HEp-2 cells," Clinical and Developmental Immunology, vol. 2012, Article ID 651058, 7 pages, 2012.

[33] S. Kivity, B. Gilburd, N. Agmon-Levin et al., "A novel automated indirect immunofluorescence autoantibody evaluation," Clinical Rheumatology, vol. 31, no. 3, pp. 503-509, 2012.

[34] A. Melegari, C. Bonaguri, A. Russo, B. Luisita, T. Trenti, and G. Lippi, "A comparative study on the reliability of an automated system for the evaluation of cell-based indirect immunofluorescence," Autoimmunity Reviews, vol. 11, no. 10, pp. 713-716, 2012.

[35] C. Bonroy, C. Verfaillie, V. Smith et al., "Automated indirect immunofluorescence antinuclear antibody analysis is a standardized alternative for visual microscope interpretation," Clinical Chemistry and Laboratory Medicine, vol. 51, no. 9, pp. 1771-1779, 2013.

[36] X. Bossuyt, S. Cooreman, H. de Baere et al., "Detection of antinuclear antibodies by automated indirect immunofluorescence analysis," Clinica Chimica Acta, vol. 415, pp. 101-106, 2013.

[37] S. S. Copple, T. D. Jaskowski, R. Giles, and H. R. Hill, "Interpretation of ANA indirect immunofluorescence test outside the darkroom using NOVA view compared to manual microscopy," Journal of Immunology Research, vol. 2014, Article ID 149316, 7 pages, 2014.

[38] R. Tozzoli, A. Antico, B. Porcelli, and D. Bassetti, "Automation in indirect immunofluorescence testing: a new step in the evolution of the autoimmunology laboratory," Autoimmunity Highlights, vol. 3, no. 2, pp. 59-65, 2012.

[39] R. Tozzoli, C. Bonaguri, A. Melegari, A. Antico, D. Bassetti, and N. Bizzaro, "Current state of diagnostic technologies in the autoimmunology laboratory," Clinical Chemistry and Laboratory Medicine, vol. 51, no. 1, pp. 129-138, 2013.

[40] N. Bizzaro, A. Antico, S. Platzgummer et al., "Automated antinuclear immunofluorescence antibody screening: a comparative study of six computer-aided diagnostic systems," Autoimmunity Reviews, vol. 13, no. 3, pp. 292-298, 2014.

[41] P. L. Meroni, N. Bizzaro, I. Cavazzana, M. O. Borghi, and A. Tincani, "Automated tests of ANA immunofluorescence as throughput autoantibody detection technology: strengths and limitations," BMC Medicine, vol. 12, no. 1, article 38, 2014.

[42] C. Krause, K. Ens, K. Fechner et al., "EUROPattern-Suite technology for computer-aided immunofluorescence microscopy in autoantibody diagnostics," Lupus, vol. 24, no. 4-5, pp. 516-529, 2015.
[43] P. Soda, L. Onofri, and G. Iannello, "A decision support system for Crithidia Luciliae image classification," Artificial Intelligence in Medicine, vol. 51, no. 1, pp. 67-74, 2011.

[44] F. Buzzulini, A. Rigon, P. Soda et al., "The classification of Crithidia luciliae immunofluorescence test (CLIFT) using a novel automated system," Arthritis Research and Therapy, vol. 16, no. 2, article R71, 2014. 


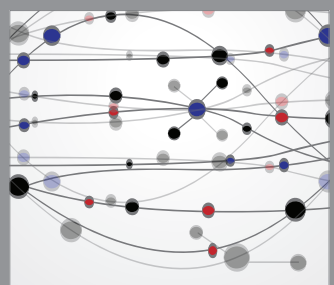

The Scientific World Journal
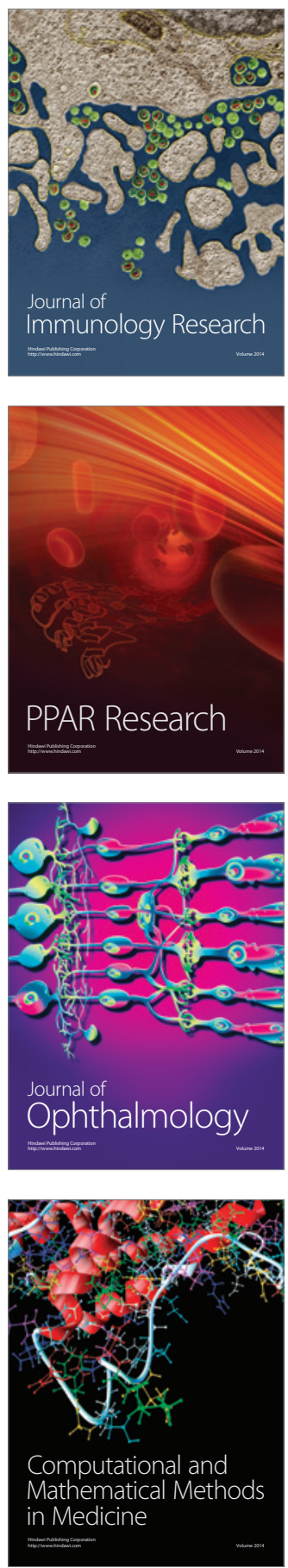

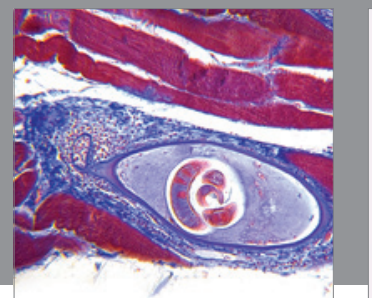

Gastroenterology

Research and Practice
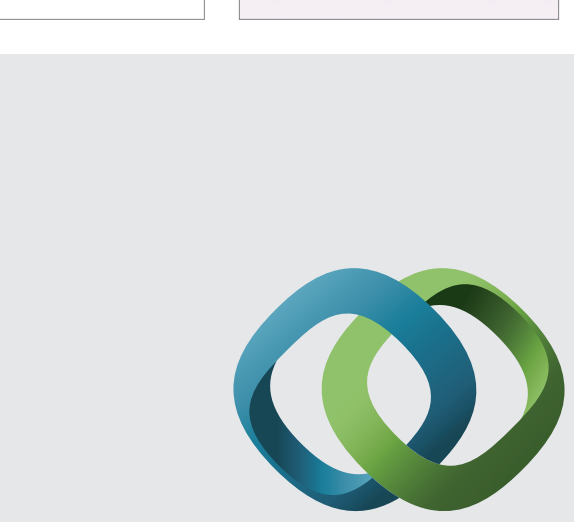

\section{Hindawi}

Submit your manuscripts at

http://www.hindawi.com
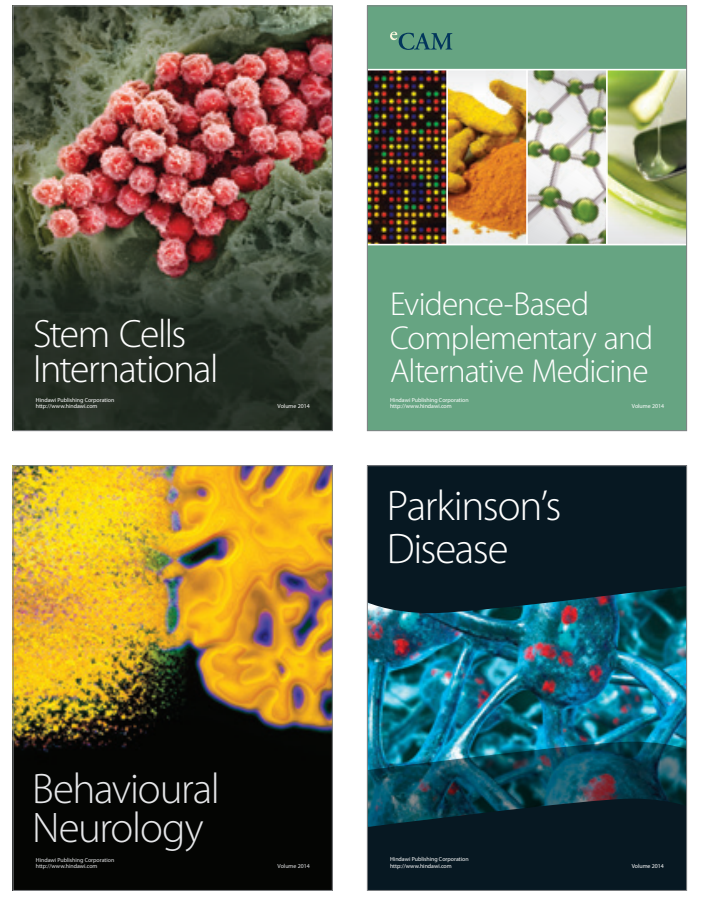
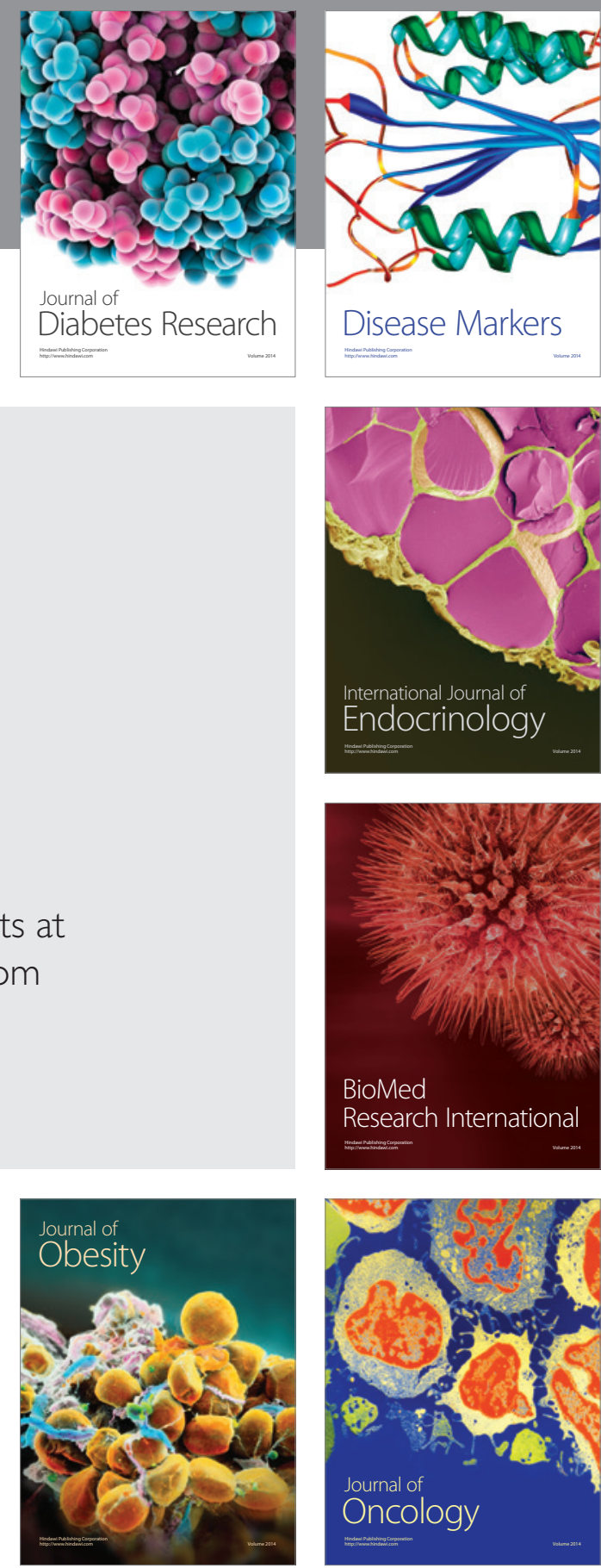

Disease Markers
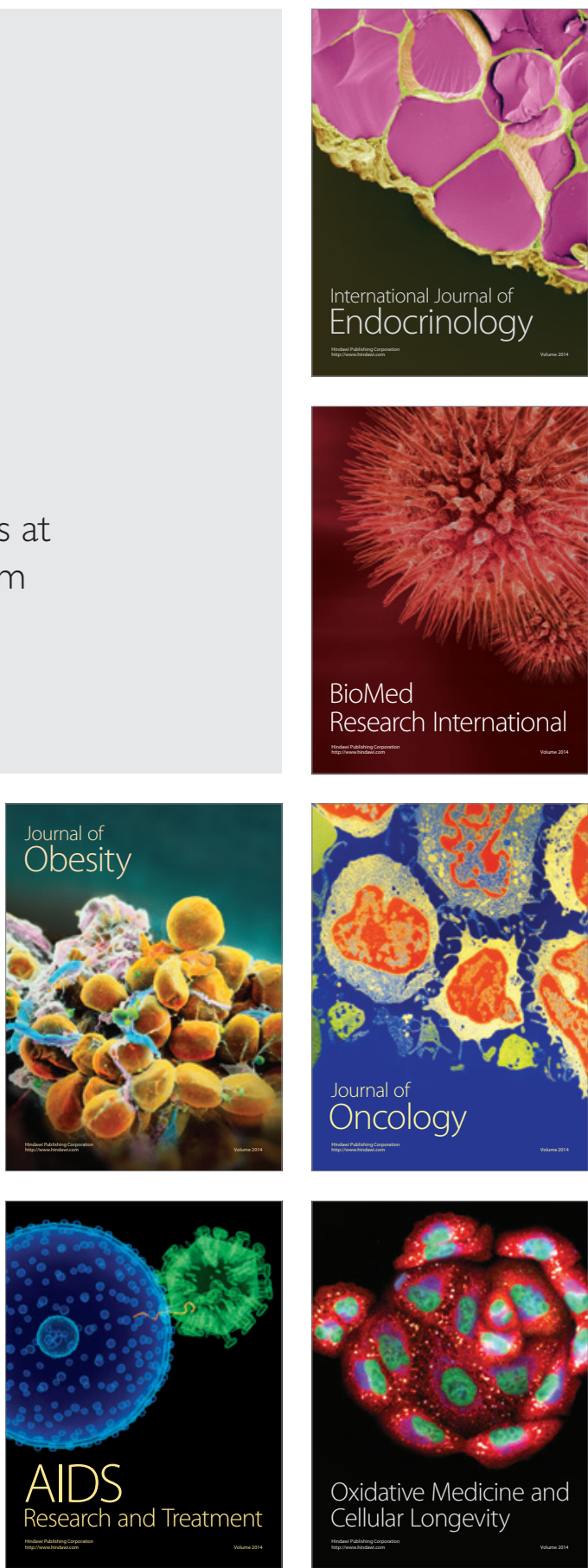